\title{
Efecto de la radiación ultravioleta (UV) en animales domésticos.
} Revisión

\author{
Maricela Olarte Saucedo ${ }^{a^{*}}$ \\ Sergio Hugo Sánchez Rodríguez ${ }^{\mathrm{b}}$ \\ Carlos Fernando Aréchiga Flores ${ }^{\text {a }}$ \\ Rómulo Bañuelos Valenzuela ${ }^{a}$ \\ María Argelia López Luna ${ }^{c}$
}

a Universidad Autónoma de Zacatecas. Unidad Académica de Medicina Veterinaria y Zootecnia. Zacatecas, México.

b Universidad Autónoma de Zacatecas. Unidad Académica de Ciencias Biológicas, Zacatecas, México.

c Universidad Autónoma de Zacatecas. Unidad Académica de Ciencias Químicas. Zacatecas, México.

* Autor de correspondencia: olarte61@ hotmail.com

\section{Resumen:}

La luz solar es necesaria para todos los organismos vivos que habitan el planeta Tierra, pero debido a la contaminación ambiental, se ha generado un cambio climático a nivel mundial, que ha afectado a los seres vivos debido al desgaste en la capa de ozono, la cual es importante para evitar el paso de la radiación ultravioleta (UV); ésta afecta principalmente a los animales domésticos que están en contacto directo con ella, provocándoles lesiones cutáneas, tumoraciones ópticas, estrés térmico o incluso la muerte. La luz UV produce en la piel estrés oxidativo, el cual se da por una excesiva producción de especies reactivas del oxígeno (ERO), que pueden dañar a la célula causando envejecimiento celular o cáncer. Los antioxidantes neutralizan a estos agentes lesivos, pero van disminuyendo su actividad con la edad y el estado metabólico del organismo. Para la realización de este trabajo se hizo una revisión sistemática en bases bibliográficas (PubMed/MEDLINE, Science) y en revistas públicas por medio de 
internet; considerando la histología y fisiología de la piel y las afecciones a ésta provocadas por la exposición a la luz UV en animales domésticos. Es importante conocer los efectos de la radiación UV en la salud de los animales domésticos, ya que puede afectar económicamente a la actividad agropecuaria, comprometer el bienestar animal y la calidad e inocuidad de los productos de origen animal.

Palabras clave: Radiación ultravioleta, Animales de granja, Piel, Cáncer.

Recibido: 30/09/2017

Aceptado: 16/04/2018

\section{Introducción}

La energía solar no solo es necesaria para la vida de los seres vivos, sino también para los animales; pero debido al cambio climático, el calentamiento global, emisión de gases y el efecto invernadero, sobre todo este último, ha generado alteraciones en la capa de ozono $^{(1,2)}$, provocando la entrada directa de la radiación UV a la superficie terrestre, ocasionando en los últimos años alteraciones al medio ambiente y a las especies animales que están en contacto directo con la radiación solar, como animales de granja, provocándoles lesiones cutáneas, tumoraciones ópticas, estrés calórico o incluso la muerte, dando como resultado grandes pérdidas económicas en el ramo agropecuario ${ }^{(3)}$.

\section{Tipos de radiación}

La radiación puede definirse como la energía que transita de un lugar a otro ${ }^{(4)}$. También se le llama radiación a toda energía que se propaga en forma de onda o de partícula a través del espacio ${ }^{(4)}$. El sol es una fuente natural de radiaciones electromagnéticas que se caracterizan por su frecuencia y longitud de onda, y suelen clasificarse en base a dos criterios:

1) Según su naturaleza:

La radiación propagada en forma de ondas (rayos gamma, rayos $\mathrm{X}$ ), radiaciones ultravioletas tipos A, B y C (UVA, UVB, UVC), radiación visible (violeta, azul, verde, amarilla, naranja, roja), radiaciones infrarrojas, radiofrecuencias (radar, microondas), se les llama radiaciones electromagnéticas ${ }^{(5,6)}$, mientras que la llamada en forma de partículas subatómicas (partículas $\alpha$, partículas $\beta$, neutrones, radiaciones cósmicas) se les 
llama radiaciones corpusculares, que se mueven a gran velocidad, con apreciable transporte de energía ${ }^{(4,5,6)}$.

2) Según su efecto biológico:

Si la radiación transporta energía suficiente como para provocar ionización en el medio que atraviesa, se dice que es una radiación ionizante. En caso contrario se habla de radiación no ionizante, la cual no puede separar electrones de los átomos o alterar las estructuras moleculares ${ }^{(7)}$, aunque la energía fotónica es débil para romper enlaces químicos, tiene efectos biológicos como son el calentamiento y la inducción de corrientes eléctricas en los tejidos y células ${ }^{(8)}$. El carácter ionizante o no ionizante es independiente de su naturaleza corpuscular u ondulatoria ${ }^{(9)}$. Son radiaciones ionizantes: radiaciones alfa, beta, rayos cósmicos, rayos gamma, rayos X, y parte del espectro de la radiación UV entre otros. Por otro lado, radiaciones como los rayos UV, visible e infrarrojo y las ondas de radio, TV o de telefonía móvil, son algunos ejemplos de radiaciones no ionizantes como se muestra en la Figura $1^{(7,8,9)}$.

Figura 1: Espectro electromagnético. Muestra las diferentes longitudes de onda que emite el sol, mediante radiaciones electromagnéticas ${ }^{(10)}$

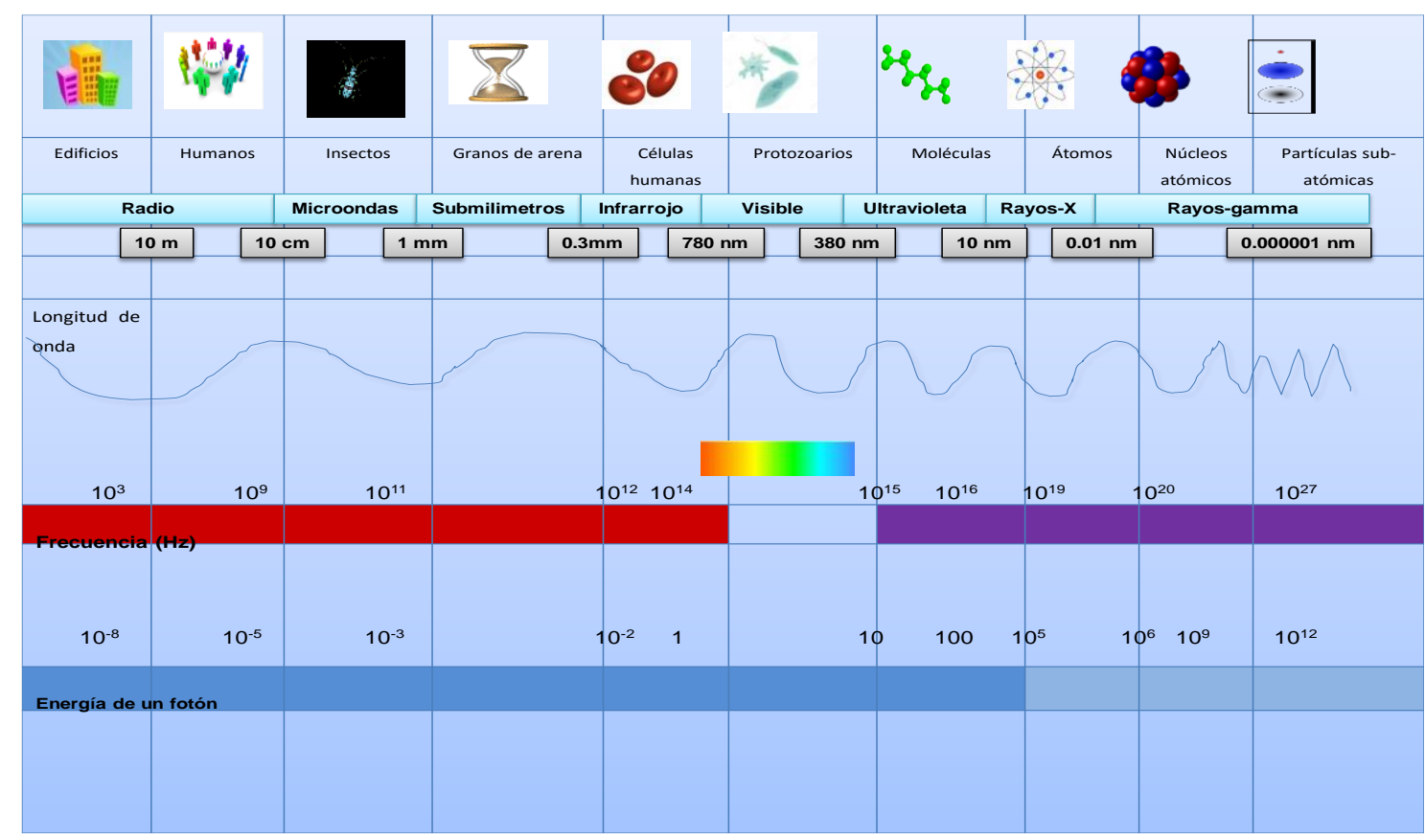




\section{Luz ultravioleta (UV)}

De todo el espectro solar sólo la luz visible, los infrarrojos y una parte de la radiación ultravioleta alcanzan la superficie terrestre, en las siguientes proporciones: 50, 40 y $10 \%$ respectivamente. El resto, son detenidas por el ozono estratosférico. La radiación solar ultravioleta, se define como la potencia de la energía solar UV por unidad de superficie (UV) y se mide en $\left(\mathrm{w} / \mathrm{m}^{2}\right)^{(11)}$, posee tres diferentes longitudes de onda: la UVA (315- 400 $\mathrm{nm})$, la UVB (280-315 nm) y la UVC (100-280 nm $)^{(4,12,13,14)}$. La UVC posee la más alta energía, pero es absorbida por la capa de ozono en la atmósfera y no tiene efectos adversos en la piel, mientras dicha capa permanezca intacta. Sin embargo, el daño creciente a la capa de ozono pudiera generar efectos nocivos de la UVC.

Las UVA y UVB se consideran un factor de riesgo en el desarrollo de cáncer de piel y llegan a la superficie de la tierra 95 y $5 \%$, respectivamente. Las UVB están implicadas en la formación de foto-productos y demás complejos, que deterioran los ácidos nucleicos con consecuencias a largo plazo, directamente relacionadas con diversas neoplasias de piel, provocadas por las quemaduras repetidas o frecuentes sobre la epidermis ${ }^{(15)}$. Por su parte, la citotoxicidad de las UVA es principalmente mediada por moléculas endógenas foto-sensibilizadoras, que absorben fotones y generan especies reactivas del oxígeno, generando daño directo sobre la dermis y el envejecimiento prematuro ${ }^{(13,15)}$.

\section{La atmósfera}

La atmósfera está compuesta en mayor cantidad por nitrógeno (78 \%) y en segundo lugar por oxígeno $(26 \%)^{(16,17)}$. El porcentaje restante corresponde a numerosos gases traza, entre los cuales se encuentra el ozono, en cantidades ínfimas de pocas moléculas por millón de partículas de aire $(0.01 \%)$. Sin embargo, cumple un rol esencial en la conservación de la vida en el planeta tal como la conocemos, ya que nos protege de la UV que es el carcinógeno físico más importante para el hombre, animales terrestres y $\operatorname{marinos}^{(16,17)}$.

El ozono es una molécula formada por tres átomos de oxígeno y se crea en dos lugares de la atmósfera, el $90 \%$ o más del ozono se produce en la parte alta de la estratósfera, a $50 \mathrm{~km}$ de la superficie terrestre y corresponde al ozono benéfico, protector de la radiación ultravioleta $^{(16)}$. Cabe mencionar que $10 \%$ del ozono se produce en las grandes ciudades, a nivel de la superficie terrestre o tropósfera, y es un componente del $\operatorname{smog}^{(16,18)}$.

En las últimas décadas, el hombre ha alterado el equilibrio ecológico de la capa de ozono con la producción y emisión a la atmósfera de las llamadas "sustancias depletoras de ozono" (SDO) ${ }^{(1,19)}$. Las más conocidas son los clorofluorocarbonos (CFC), que se usaron 
en la fabricación de aerosoles, refrigeradores y equipos de aire acondicionado, estos CFC son muy reactivos, una molécula de cloro puede destruir mil moléculas de ozono ${ }^{(1,19)}$. Al aumentar en la atmósfera los compuestos que degradan el ozono, como su velocidad de formación es lenta, su concentración disminuye hasta que se alcance un nuevo equilibrio entre la velocidad de formación y la degradación ${ }^{(16,17,19)}$.

La radiación solar es uno de los principales factores ambientales que afectan la vida en nuestro planeta. Esta radiación controla el funcionamiento de los ecosistemas terrestres y acuáticos a través del control de procesos fotobiológicos (fotosíntesis, fotoperiodo, fototropismos) así como de su acción sobre otros factores ambientales como la temperatura, humedad y ciclos naturales (ciclos diarios, anuales, hídricos), que finalmente inciden en la distribución de los organismos ${ }^{(19,20)}$.

La radiación que llega a la Tierra abarca una amplia gama del espectro electromagnético y aproximadamente el $40 \%$ de ella es la que conocemos como luz o radiación visible. Esta comprende longitudes de onda que van de los 400 a los $700 \mathrm{~nm}$, y que es usado por los vegetales en el proceso de la fotosíntesis. Otro rango de esta radiación electromagnética es el que va de 280 a $1,000 \mathrm{~nm}$, conocido como rango fotobiológico ${ }^{(21)}$.

La cantidad y calidad de las radiaciones que llegan a la Tierra depende tanto de la energía solar emitida como de las características de la atmósfera en un sitio dado.

La luz UVA y UVB penetran en la biósfera, pero solo la UVB es absorbida por el ozono atmosférico, por lo que la cantidad que alcanza la superficie terrestre aumenta como resultado de la disminución de este gas. Sólo el $1.3 \%$ de la luz ultravioleta emitida por el sol alcanza la superficie de la tierra, de ese porcentaje, el $98 \%$ corresponde a la UVA y el $2 \%$ a la UVB, mientras el resto de la luz ultravioleta es fuertemente absorbida en la atmósfera.

Esta misma radiación solar, la cual ha hecho posible la vida sobre nuestro planeta, puede ser perjudicial en altas intensidades o cuando la proporción de ondas cortas aumenta sobre determinados límites. La radiación de alta intensidad y los cambios en la composición espectral pueden afectar importantes procesos en los organismos ${ }^{(19,21)}$.

\section{Fisiología de la piel en animales}

La piel, conforma la superficie del cuerpo que establece relación directa con el medio ambiente, está constituida por tres estratos o capas que presentan a la vez un conjunto de estructuras anexas como glándulas sudoríparas y sebáceas ${ }^{(22)}$. Existen diferentes formas de protección corporal según la especie animal (pelos, lana, plumas) y partes queratinizadas (uñas, cascos y pezuñas). La piel desempeña diferentes funciones como son: órgano protector contra estímulos mecánicos, físicos y químicos del medio ambiente 
que agreden la integridad del cuerpo animal ${ }^{(22,23)}$. Aumenta su espesor en aquellos puntos que se encuentran sometidos regularmente a compresiones mecánicas (pezuñas, cascos, almohadillas plantares y pulpejos) ${ }^{(22)}$. La piel proporciona también protección contra radiaciones $^{(24)}$, principalmente radiaciones solares, de diferentes longitudes de ondas, de ahí que en su estrato superficial o epidermis, en muchas especies de animales, se formen pigmentos (gránulos de melanina) que impiden la penetración de las radiaciones a los tejidos profundos tal como se observa en la piel del oso polar que, como adaptación a la fuerte intensidad luminosa por acción directa de los rayos solares e indirecta por ser reflejados por el hielo o la nieve, presenta pelaje blanco (refractario) con piel negra (protectora) ${ }^{(24)}$. La piel es relativamente impermeable a microorganismos y a muchas sustancias venenosas y nocivas al cuerpo animal.

La presencia en la piel de glándulas sudoríparas y sebáceas, cuyas secreciones son vertidas por los conductos glandulares al exterior, le confiere a este tejido un papel excretor $^{(24)}$. La pérdida de agua por la piel constituye una vía de termorregulación no asociada al mantenimiento del equilibrio hídrico, pero sí, asociada a las condiciones térmicas de la relación entre el animal y el medio ambiente. El sebo cutáneo, producto de naturaleza grasosa secretado por las glándulas sebáceas, protege a la piel contra la humedad y le confiere suavidad y textura ${ }^{(24)}$.

La piel desarrolla un importante papel en el sistema de crecimiento o desarrollo corporal somático en el cuerpo de los animales, al constituirse en el área de almacenamiento y activación primaria de la vitamina $\mathrm{D}^{(23)}$. La vitamina $\mathrm{D}$ que ingresa al organismo en forma de D2 (ergocalciferol) o D3 (colecalciferol) según la fuente de ingreso, por circulación sanguínea alcanza la piel, donde se almacena en forma de calciferol o precursor, y por acción de los rayos ultravioleta del sol se transforma en colecalciferol, que al alcanzar de nuevo la circulación sistémica pasa primero al hígado y finalmente a los riñones, en donde por efecto de la parathormona $(\mathrm{PTH})$ se convertirá en la vitamina D hormona $(1,25$,dihidroxicolecalciferol), que desarrolla su función a nivel de la mucosa intestinal evitando el raquitismo del animal al estimular la absorción facultativa del calcio ${ }^{(22)}$.

\section{Histología de la piel}

El área cutánea total depende de la especie animal, calculándose, por ejemplo, que en personas adultas puede alcanzar hasta los $2 \mathrm{~m}^{2}$; en ciertos territorios cutáneos, dependiendo de la especie animal, se desarrollan formaciones apendiculares especiales como pelos o plumas, uñas, cuernos, cascos o pezuñas, así como numerosas, escasas o ausencia de glándulas sudoríparas y sebáceas ${ }^{(25)}$.

El grosor de la piel es variable, en general es más gruesa en la superficie dorsal del cuerpo y en las caras laterales de las extremidades, más delgada en la cara ventral del cuerpo y 
caras mediales de las extremidades, existiendo diferencias en las zonas relacionadas con el sexo, la raza y la especie ${ }^{(22)}$. La media de las zonas más delgadas oscila entre los 0.4 $\mathrm{mm}$ en el murino hasta los $2.4 \mathrm{~mm}$ en el bovino Holstein (Bos taurus), raza productora de leche, mientras que en las zonas gruesas este valor comprende desde los $1.9 \mathrm{~mm}$ en el gato hasta los $10.7 \mathrm{~mm}$ en el garañón o caballo semental ${ }^{(24)}$.

En la piel se distinguen tres estratos: epidermis, parte epitelial o estrato de superficie, dermis, parte conjuntiva o estrato intermedio profundo, e hipodermis o tejido celular subcutáneo (Figura 2) $)^{(25,26)}$.

Figura 2: Capas de la piel gruesa de rata

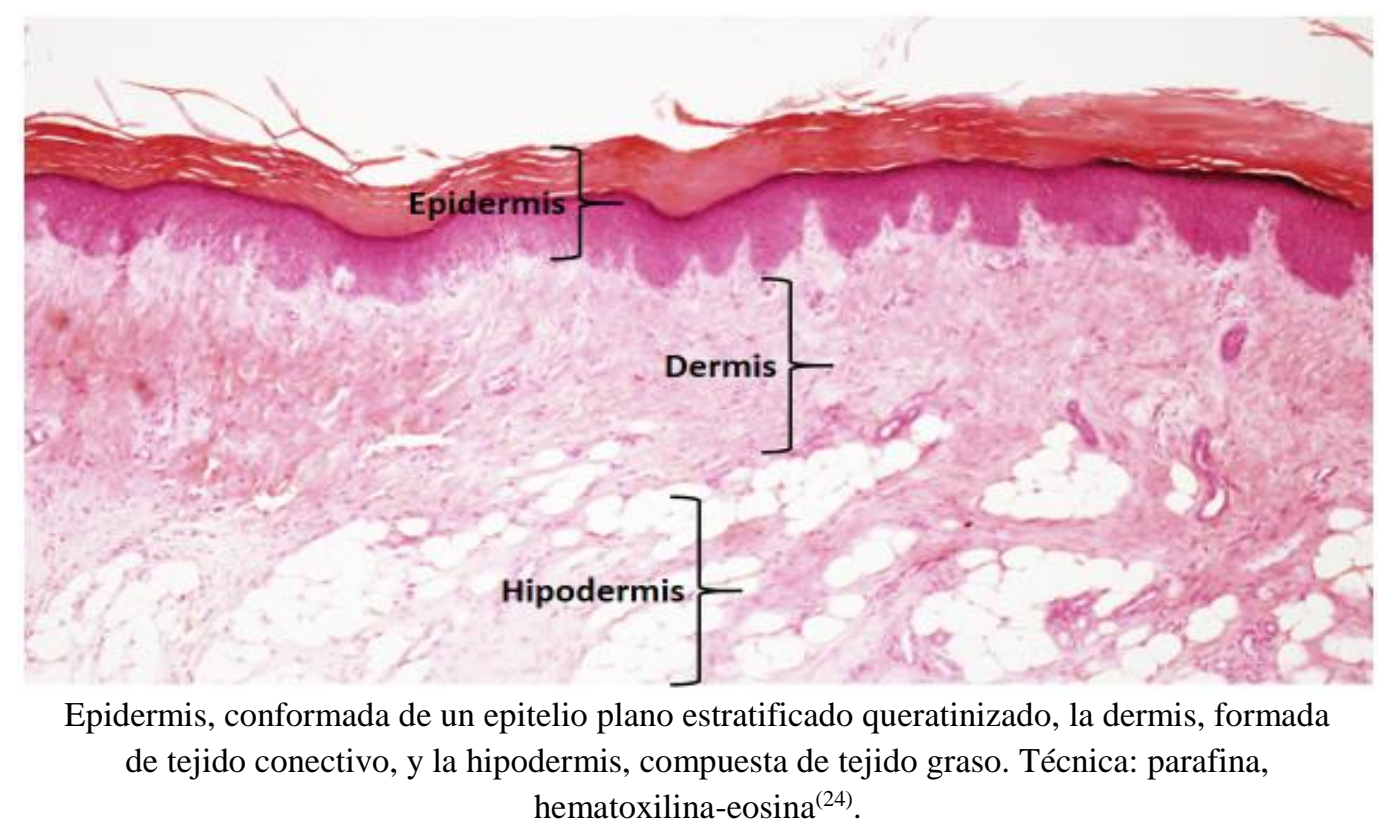

Epidermis. Está constituida por un epitelio plano estratificado queratinizado, y se divide en estratos: estrato germinativo, estrato espinoso, estrato granuloso, estrato lúcido y estrato córneo ${ }^{(25,26,27)}$.

Dermis. Se pueden distinguir dos capas: capa papilar, en posición más superficial y capa reticular, en posición más profunda. La capa papilar o superficial de la dermis situada inmediatamente por debajo de la epidermis, consta de una trama densa de tejido conjuntivo irregular fibroso laxo, que cumple una función trófica y recibe su nombre por las numerosas papilas que se proyectan sobre la epidermis; es más ancha en la piel del caballo y vacunos que en los carnívoros ${ }^{(25-28)}$.

Hipodermis. Es una capa de tejido conjuntivo que fija la piel a los huesos y a los músculos. La función primaria de esta capa es la amortiguación a las presiones externas y permitir el movimiento libre sobre las estructuras subyacentes. En este estrato también 
se halla presente una capa de tejido adiposo que puede adoptar la forma de pequeños grupos de células, o de grandes masas que dan lugar a la formación de las almohadillas o cojines de grasa, cuya función es termorreguladora para los animales que viven en climas templados al aumentar su grosor en el invierno, y servir como un aislante térmico retenedor del calor ${ }^{(24,25)}$. Por otra parte, los cascos, las pezuñas, las uñas, los cuernos, los espolones y las espuelas son estructuras que tienen su origen en procesos de queratinización del estrato córneo con espesor y consistencia diferente ${ }^{(24,25)}$.

Pelos. Los pelos son formaciones epidérmicas extendidas en la mayoría de los mamíferos por toda la piel excepto en las almohadillas plantares, los cascos, las uñas, parte de los labios, el glande, la parte interna del prepucio, los labios vulvares, los pezones y la cara plantar de las extremidades. El pelo consta de raíz, tallo y la punta que es la parte que sobresale de la piel. Las raíces de los pelos están rodeadas por una invaginación de los estratos espinoso y germinativo de la epidermis, que se introducen en la dermis, de manera que al situarse en el estrato papilar de ésta se relacionan con los vasos sanguíneos ${ }^{(25)}$.

La proporción existente entre la capa cortical y medular del pelo depende de la especie animal, y así vemos que el pelo que constituye el revestimiento piloso en el caballo, la vaca, el perro y el cerdo, posee una capa cortical más gruesa que el pelo de revestimiento en la cabra y el gato. Los pelos finamente rizados de las ovejas y cerdos, en los erizos o el puercoespín, se manifiestan como pelos afilados conocidos como espinas o púas, en los animales jóvenes poseen vello y prácticamente carecen de médula ${ }^{(24,25,28,29,30)}$.

\section{Adaptaciones de la piel en respuesta a las condiciones ambientales}

La adaptación morfo-fisiológica de la piel animal a las condiciones del medio ambiente de carácter evolutivo, involucra las particularidades morfológicas de la piel y su capacidad para permitir ajustes térmicos a las variables ambientales, para facilitar el incremento o disminución de las pérdidas de calor ${ }^{(28)}$. Estudios histológicos comparados entre ganado bovino Cebú (Bos indicus), con giba y el Holstein (Bos taurus), muestran que el grosor de la piel no es homogéneo en toda la superficie corporal al compararse las mismas zonas entre animales de la misma especie, pero de razas diferentes, e inclusive, la edad también determina cambios de grosor en áreas cutáneas; el estudio comparativo de 21 regiones del cuerpo en donde se midió el pliegue cutáneo en la raza HolsteinFriesian (Bos taurus) demostró cambios de grosor en una misma área, e incrementó el grosor general de la piel a medida que aumenta la edad del animal. El análisis comparativo entre diferentes estructuras macro y microscópicas de la piel en hembras bovinas de las razas Holstein y Cebú explica la mejor adaptación del Bos indicus (Cebú) a las altas temperaturas ${ }^{(28,31)}$, donde presenta un pelo más corto y grueso, un mayor grosor de la piel con epidermis más delgada y una dermis reticular más profunda, un mayor volumen de 
glándulas sudoríparas con implantación dérmica, por lo que posee una mayor superficie excretora y una densidad glandular incrementada por área ${ }^{(28)}$.

La raza bovina Holstein-Friesian, una raza en la producción de leche, se caracteriza por tener una piel de menor grosor, epidermis más gruesa y dermis reticular más fina. Es interesante señalar que mientras en la raza Holstein las glándulas sudoríparas tienen forma tubular con diferentes grados de torsión, en el Cebú las mismas se presentan de forma sacular, con un alto grado de concentración por área, lo que asegura un sistema disipador de calor capaz de permitir la respuesta de adaptación a las altas temperaturas ambientales del trópico ${ }^{(28,32)}$.

\section{Afectación de la radiación solar a los animales}

Se ha observado que los animales que están expuestos por largos periodos de radiación solar, que viven a grandes altitudes sobre el nivel del mar y en lugares tropicales, carecen de pigmento en la epidermis, tienen poco pelo o pérdida del mismo, y son más propensos a enfermedades de la piel ${ }^{(33-36)}$, debido a que la luz ultravioleta daña el ácido desoxirribonucleico $(\mathrm{ADN})$ de la célula ${ }^{(32)}$, induce los dímeros de pirimidina ciclobutano (CPD), pirimidina $(6,4)$ y pirimidonina $(6,4 \mathrm{PP})$ que causan efectos deletéreos como la inhibición de la replicación y de la transcripción, el aumento en la aparición de mutaciones, la detención del ciclo celular y la muerte celular ${ }^{(37)}$. Uno de los padecimientos que se relaciona con estos factores es el carcinoma de células escamosas (CCE), también conocido como carcinoma de células espinosas o espinocelular o carcinoma epidermoide $^{(34)}$, el cual es un tumor maligno que afecta a los queratinocitos de la epidermis de la piel ${ }^{(35,36)}$, es localmente invasivo, no necesariamente metastásico ${ }^{(33)}$, pero puede comprometer la dermis ${ }^{(38)}$.

Estos tumores se encuentran principalmente en bovinos, afectando principalmente a las razas Hereford, Simmental, y Holstein, las cuales poseen piel blanca y sin pigmentación, especialmente, en los ojos ${ }^{(34,35)}$, por lo que se tienen pérdidas millonarias al año, debido a cáncer de ojo ${ }^{(39)}$, enfermedad conocida como ojo rosado o "pink eye", el cual es común en este tipo de animales. Afecta a los más viejos pero no excluye a jóvenes, principalmente de cara blanca y poco pigmentada; es de origen genético, pero también se relaciona con la exposición a la radiación ultravioleta ${ }^{(38)}$. Sin embargo, también afecta a felinos y canideos ${ }^{(40,41)}$, poco común en ovinos y raro en caprinos y porcinos ${ }^{(33,35,36)}$. En caballos, las razas más sensibles son Belga, Clydesdale, Shire y Appaloosa ${ }^{(34)}$. La aparición de las lesiones en estos animales es principalmente en regiones muco-cutáneas (conjuntiva, vulva, perineo) $^{(34)}$. En canideos, su frecuencia es de un 20 a $30 \%$ y en felinos es de $70 \%{ }^{(42)}$; no hay una diferencia entre sexo, aparece en razas grandes y animales mayores a 10 años $^{(42)}$. En canideos las lesiones están localizadas principalmente en 
tronco, extremidades, escroto, labios, y el lecho ungular ${ }^{(38)}$, en felinos, en cara, orejas y principalmente en gatos de pelo blanco ${ }^{(42)}$.

Otra patología relacionada con la exposición a la luz ultravioleta, son los melanocitomas, los cuales se forman a partir de las células encargadas de dar pigmentación a la piel, pestañas y pelo llamadas melanocitos, ubicados en la epidermis de la piel ${ }^{(43)}$. El 80 al $90 \%$ de estos tumores son benignos en bovinos, localizándose principalmente en piel ${ }^{(43)}$; este padecimiento afecta en todas las edades, no hay predisposición por el sexo, aqueja a ganado de color obscuro (gris, rojo y negro) ${ }^{(43)}$; dichos tumores aparecen en cualquier parte principalmente en las extremidades ${ }^{(43)}$. En el resto de los animales estos tumores suelen ser malignos y son llamados melanomas, siendo comunes en caninos y equinos, poco comunes en gatos y raros en otras especies ${ }^{(41,43,44)}$.

Los melanomas suponen el $4.7 \%$ del total de neoplasias y más del $7 \%$ de los tumores malignos en el perro ${ }^{(44,45)}$. Las localizaciones más habituales incluyen la boca (56 \%), labios $(23 \%)$, piel $(11 \%)$, dedos $(8 \%)$ y otras localizaciones $(2 \%)$ incluyendo el ojo ${ }^{(46)}$. Los melanomas cutáneos son también relativamente frecuentes. Sin embargo, del conjunto de melanomas malignos, sólo un $10 \%$ son cutáneos, con cierta predilección por la región de la cabeza y el escroto.

La incidencia de melanoma en caninos no sólo varía con la localización, sino también con la raza. Es más frecuente en razas con marcada pigmentación cutánea, como el Schnauzer o el Scottish Terrier ${ }^{(45,46)}$. El Setter Irlandés y el Golden Retriever presentan mayor incidencia de melanomas subungueales. El Setter Irlandés, el Chihuahua, Golden Retriever y el Cocker Spaniel presentan mayor riesgo para la localización labial ${ }^{(45,46)}$. Finalmente, el Pastor Alemán y el Bóxer muestran mayor riesgo de desarrollo de melanomas orales ${ }^{(47,48)}$. La edad en la que se presenta oscila entre 1 y 17 años, la media está en 10. Al igual que en personas, se establece una mayor incidencia en machos que en hembras ${ }^{(47)}$.

El melanoma en el gato es infrecuente (menos del $1 \%$ de las neoplasias orales y cerca del $0.5 \%$ de las neoplasias cutáneas $)^{(49,50,51)}$. La localización ocular y cutánea es más frecuente que la intraoral ${ }^{(52,53)}$. La localización cutánea más habitual es la cabeza, la cola, la zona distal de las extremidades y la zona lumbar ${ }^{(46,53,54)}$. El pronóstico es con frecuencia pobre, dado que la mitad de los casos muestran recurrencia y metástasis regional ${ }^{(46,54,55)}$. El rango de edad de los animales afectados es de 2 a 18 años, con un pico entre los 8 y 12 años ${ }^{(54,55)}$. No parece haber predilección de sexo o raza $^{(54,55)}$.

Otros padecimientos provocado por este factor son los hemangiosarcomas, tumores malignos los cuales afectan más comúnmente a perros de mediana edad y tercera edad, en especial a perros de raza grande, como el galgo; afecta sobre todo el bazo, el atrio derecho, el tejido subcutáneo/dérmico y el hígado ${ }^{(56)}$. También los hemangiomas se relacionan con la luz UV, es una neoplasia relativamente benigna de los capilares caninos en la piel en el tronco y las extremidades y los tejidos blandos y, con frecuencia, precursores de los hemangiosarcomas ${ }^{(57)}$. 
De tal forma que la radiación ultravioleta afecta a algunas especies de manera importante tanto en aspectos de salud, como en aspectos de trascendencia económica.

\section{Efectos patológicos de la radiación ultravioleta}

Las radiaciones UVA son capaces de inducir eritema, pigmentación inmediata o retardada, alteraciones del tejido conectivo dérmico, liberación de mediadores vasoactivos, y que favorecen el estrés fotooxidativo. Incluso, pueden incrementar el eritema por UVB, así como la carcinogénesis y la elastosis por UVB, causando alteraciones en el $\mathrm{ADN}^{(58)}$ y otras estructuras, como las fibras elásticas; son responsables de muchas reacciones de fotosensibilidad a drogas, y juegan un papel significativo en enfermedades tales como erupción polimórfica a la luz, dermatitis actínica crónica, reticuloidosis actínica, lupus eritematoso, urticaria solar, reacción persistente a la luz y xero-dermapigmentosum $(\mathrm{XP})^{(59,60)}$. La fotosensibilidad en animales se clasifica en tres tipos principales: 1) Tipo I ó primaria; este tipo de fotosensibilización es causada por compuestos fluorescentes que son depositados inalterados sobre la piel luego de la ingestión, siendo el hígado normal, incapaz de excretar el compuesto fluorescente original; ejemplos de dichas sustancias fotosensibilizantes incluyen a la hipericina, la fagopirina y productos químicos como la fenotiacina (sulfóxido de fenotiacina). 2) Tipo II o síntesis anormal de pigmento endógeno o porfiria congénita; este tipo de fotosensibilización se debe a la acumulación de pigmentos endógenos del metabolismo anormal de la porfirina. Los agentes fotodinámicos incluyen a la uroporfirina I, coproporfirina I y protoprorfirina III. Estos se acumulan en la sangre y los tejidos cuando existe una disfunción en la biosíntesis del grupo hemo, debido a una deficiencia enzimática. Por ejemplo, la porfirina eritropoyética congénita de los bovinos es causada por la deficiencia de uroporfirinógeno III cosintetasa, una enzima clave en la biosíntesis del grupo hemo. y 3) Tipo III ó fotosensibilidad hepatotóxica, la cual es más común y de relevancia económica, debido a que los animales son sensibilizados por la acumulación de filoeritrina, un producto de la digestión de la clorofila en la circulación periférica. La filoeritrina normalmente es excretada en la bilis por el hígado, pero en ciertos tipos de lesiones difusas, el hígado se asocia con una variedad de hepatotoxinas vegetales, fungales y químicas que se absorben gradualmente por el sistema circulatorio hasta que se alcanzan niveles que generan la fotosensibilidad. La materia tóxica actúa directamente sobre las células del hígado y las células de los pequeños conductos biliares, haciendo que se estenosen y eviten el paso de la bilis, lo que origina la ictericia o color amarillento $^{(60)}$.

Existen suficientes evidencias experimentales y clínicas que establecen una relación causal estrecha entre el cáncer de piel y la exposición prolongada a la luz ultravioleta, 
fundamentalmente el melanoma maligno (MM), el carcinoma de células escamosas (CCE) y el carcinoma de células basales $(\mathrm{CCB})^{(59,60)}$.

\section{Efectos benéficos de la radiación $\mathrm{UV}$ en animales}

La radiación solar ofrece la posibilidad a los organismos homeotermos de obtener una temperatura apropiada para que puedan llevar a cabo su metabolismo ${ }^{(61)}$. La deficiencia de vitamina D mejor documentada es la exposición inadecuada a la luz solar, debido a que existe una fuerte asociación entre las UVB y el metabolismo de la misma ${ }^{(62)}$. La deficiencia de dicha vitamina genera efectos inmediatos sobre el sistema esquelético, incrementando el riesgo de fracturas, la vitamina $\mathrm{D}_{3}$, se produce diariamente para controlar la absorción, transporte y depósito de calcio y, en menor proporción, de fósforo, interviniendo directamente en el mantenimiento óseo y la regulación del crecimiento. Asimismo, es necesaria para el funcionamiento hormonal, el desarrollo de órganos y la embriogénesis ${ }^{(62)}$.

También es importante para mantener a los animales en estado saludable, proveerles la radiación UVB es necesaria para los procesos fotoquímicos involucrados en la síntesis de vitamina $\mathrm{D}^{(62)}$. De la misma manera, aun cuando los animales se encuentren recibiendo una dieta adecuada y una temperatura óptima, si no se les provee el tipo de radiación necesaria para la producción de vitamina $\mathrm{D}$, no podrán incorporar dichos minerales de manera apropiada ${ }^{(62)}$.

La irradiación proporciona importantes beneficios para la salud. Este método ayuda a mantener los alimentos de manera más segura, hace posible conservar los mismos durante más tiempo en mejores condiciones, evita que se deterioren y echen a perder o que se produzcan condiciones no deseadas como sería la aparición de tubérculos. Destruyen algunos insectos, hongos y bacterias ${ }^{(63)}$.

\section{Conclusiones}

Los animales domésticos están expuestos a la radiación ultravioleta la mayor parte del tiempo, pero debido al cambio climático, la radiación UVB afecta la piel de estos animales; algunas razas de animales son más sensibles, las cuales pueden presentar algunas patologías cutáneas, como cáncer de piel entre otras, pudiendo causar pérdidas económicas cuantiosas en el sector agropecuario, generar alteraciones en la salud y en el bienestar de los animales, así como comprometer la calidad e inocuidad de los productos de origen animal destinados al consumo humano. 


\section{Literatura citada:}

1. Toca TC. Impacto ambiental empresarial y fallas de la acción pública: una realidad de las localidades bogotanas. Argos 2011;28:244-269.

2. Albanil E, Ramírez R, Jiménez B. Reporte del Clima en México http://smn. cna.gob.mx/climatologia/analisis/reporte/Anual2014.pdf, Consultado 26 de Feb, 2015.

3. Alonso S, Ramírez N, Taylor PJ. El cambio climático y su impacto en la producción de alimentos de origen animal. http: //www.veterinaria.org/revistas/redvet/ n111112.html, Consultado 29 de May, 2015.

4. Ferramola SA, Sancovich HA. Interacciones de las radiaciones electromagnéticas y especies reactivas del oxígeno sobre la piel. Rev Argent Dermatol 2006;87:113-120.

5. Rivas AM, Rojas EE, Cortés NJ, Santander GE. Efecto de la altura en la radiación solar ultravioleta en Arica Norte de Chile. Revista Facultad de Ingeniería, U.T.A. 2002;10:59-62.

6. Bohórquez BJ, Pérez JF. Radiación ultravioleta. Ciencia \& Tecnología para la salud visual y ocular. Rev Lasalle 2007;9:97-104.

7. Navas G, Jairo T, Rodrigo C. Non-Ionizing Radiation Detection. Dyna 2009;76:7181 .

8. Guerreo AJ, Peréz AJ. Las radiaciones no ionizantes y su efecto sobre la salud humana. Rev Cubana Med Milit 2006;35:3-11.

9. Pérez A, Miranda LR. Radiaciones electromagnéticas y salud en la investigación médica. Rev Cubana Med Militar 2010;39:35-43.

10. Mignone C, Barnes R. More than meets the eye: the exotic, high-energy Universe. Sci in School 2012;24:53-58.

11. Rivas A, Rojas E, Madronich S. Aumento del índice solar ultravioleta con la altura. Rev Chilena Ing 2008;16:383-388.

12. Gonzáles PM, Vernhes TM, Sánchez LA. La radiación ultravioleta. Su efecto dañino y consecuencias para la salud humana. Theoria 2009;18:69-80.

13. Vallejo EO, Vargas N, Martínez LM, Agudelo CA, Ortiz IC. Perspectiva genética de los rayos UV y las nuevas alternativas de protección solar. http://www.scielo. org.ar/scielo.php?script=sci_arttext\&pid=S1851300X2013000300002Rev, Consultado 17 Sep, 2015.

14. Luigi PG, Rightler AL, Heronc M, Gabbuttc CD. Extending human perception of electromagneticradiation to the UV region through biologically inspired photochromic fuzzy logic (BIPFUL) systems. Chem Commun 2016;52:1474-1477. 
15. Byrne SN, Hammond KJ, Chan CY, Rogers LJ, Beaugie C, Rana S, MarshWakefield F, Thurman JM, Halliday GM. The alternative complement component factor B regulates UV-induced oedema, systemic suppression of contact and delayed hypersensitivity, and mast cell infiltration into the skin. Photochem Photobiol Sci 2015;14:801-806.

16. Sánchez C. Consideraciones sobre la capa de ozono y su relación con el cáncer de piel. Rev Méd Chile 2006;134:1185-1190.

17. Erickson DJ, Sulzberger B, Zeppc RG, Austind AT. Effects of stratospheric ozone depletion, solar UV radiation, and climate change on biogeochemical cycling: interactions and feedbacks. Photochem Photobiol 2015;14:127-148.

18. Environment Canada. The UV Index and Ozone http://www.ec.gc.ca/uv/ Default.asp?lang=En\&n=C74058DD, Accessed Feb 16, 2015.

19. Bornman JF, Barnes PW, Robinson SA, Ballaré CL, Flinte SD, Caldwell MM. Solar ultraviolet radiation and ozone depletion driven climate change: effects on terrestrial ecosystems. Photochem Photobiol 2015;14:88-10.

20. Baiz AF, McKenzie RL, Bernhard G, Aucamp PJ, Ilyas M, Madronich S, Tourpali K. Ozone depletion and climate change: impacts on UV radiation Photochem Photobiol. Sci 2015;14:19-52.

21. Carrasco RL. Efecto de la radiación ultravioleta-B en plantas. IDESIA Chile 2009;3:59-76.

22. Castellanos I, Clarena G, Rodríguez T, Iregui G. Estructura histológica normal de la piel del perro. Rev Med Vet 2005;10:104-122.

23. Vega PC. Manual de Histología Esquemática. 1ra ed. La Habana, Cuba: Editorial Pueblo y Educación; 1980.

24. Dellmann HDB. Histología veterinaria. 2a ed. España: Editorial Acribia, Zaragoza; 1994.

25. Megías M, Molist P, Pombal MA. Atlas de histología vegetal y animal. Órganos animales tegumento, https://mmegias.webs.uvigo.es/descargas/o-ategumento.pdf, Consultado 24 Feb, 2016.

26. Paniagua R, Nistal M, Sesma P, Álvarez U, Fraile M, Anadón B, Sáez RFS, De Miguel MP. Citología e histología vegetal y animal. 2a ed. España: Editorial McGrawHill-Interamericana de España; 1997.

27. Ross MH, Pawlina W. Histología Texto y Atlas. $7^{\text {a }}$ ed. Barcelona, España: Editorial Wolters Kluwer; 2016. 
28. Esquivel VC. La raza, el pelo y la piel en función del bienestar animal. Mundo Pecuario 2012;1:73-85.

29. Suro RJ, Gutiérrez FL, Ruiz AJ, Bouhann P. El pelo. Generalidades y funciones. Dermatología CMQ 2007;4:218-223.

30. William J. Applied Veterinary. 2a ed. USA: Editorial Mosby; 1993.

31. Kamwanja LA, Chase CC, Gutierrez JA, Guerriero V, Olson TA, StatusHammond AC, Hansen PJ. Responses of bovine lymphocytes to heat shock as modified by breed and antioxidan. J Anim Sci 1994;72:438-444.

32. Pérez EH. Fisiología Animal II. http://cenida.una.edu.ni/relectronicos/RENL 50P438.pdf. Consultado 28 Sep, 2016.

33. Rosolem MC, Moroz LR, Rodigheri SM. Carcinoma de células escamosas. Pubvet 2012;6:6-14.

34. Ramos AT, Mollerke ND, Elias FM, Gevehr FC. Carcinoma de células escamosas em bovinos, ovinos e eqüinos: estudo de 50 casos no sul do Rio Grande do Sul. Braz Vet Res Anim Sci 2007;44:5-13.

35. Fabricio KL, Dantas AF, Riet CF, De Miranda NE, Simões SV, Azevedo SS. Fatores de risco associados à ocorrência de carcinoma de células escamosas em ruminantes e equinos no semiárido da Paraíba. Pesq Vet Bras 2012;32:881-886.

36. Targino J, Almeida S, Macedo E, Almeida BE, Santana de OR, Anjos FE, Ocampos PPM. Squamous cell carcinoma in the frontal region of the head in a goat. Acta Scient Vet 2013 41:4-8.

37. Tafurt Y, Marin MA. Principales mecanismos de reparación de daños en la molécula de ADN. Rev Biosalud 2014;132: 95-110.

38. Costa C, Ramos VD, Huppes R, Bardoza DA, Gaspar RA, Rivera CL, Ramirez UR. Cryosurgery in the treatment of squamous cell carcinoma in dog. Rev Colomb Cienc Anim 2013;5:213-221.

39. Heather ST. Combata el cáncer de ojo. Hereford, Bs.As. 1999;(64):38-40.

40. Olinda RG, Frade MT, Soares GS, Aguiar GM, De Costa VM, Lucena RB, De Dantas AF. Bovine demodicosis associated with squamous cell carcinoma of the vulva. Acta Scient Vet 2013;41:29-32.

41. Mukaratirwaa S, Chipunzaa J, Chitangaa S, Chimonyoa M, Bhebhea E. Canine cutaneous neoplasms: prevalence and influence of age, sex and site on the presence and potential malignancy of cutaneous neoplasms in dogs from Zimbabwe. J S Afr Vet Ass 2005;76:59-62. 
42. Revista de la Asociación Madrileña de Veterinarios de Animales de Compañía. https://es.scribd.com/document/26893256/Revista-de-La-Asociacion-Madrilena-deVeterinarios, Consultada 14 Ene, 2010.

43. Chávez VC, Astaiza MJ, Benavides MC, Vallejo TD. Tumor maligno derivado de melanocitos en piel de un bovino de presentación inusual: estudio de caso. Rev Med Vet 2015;29:63-72.

44. Iakatos I, Mirela E, Cadar, Ali B. Cutaneous tumors' incidence in dog. Lucrări Stiinłifice Medicină Veterinară 2009;13:475-381.

45. Silva HG, Juárez BF, López VM, Dávila PM. Squamous cell carcinoma in dogs from Culiacan, Sinaloa, Mexico Retrospective Rev (2006-2014). Rev Cient, FCV-LUZ 2015;25:573-584.

46. Goldschmidt MH, Shofer FS. Skin tumors of the dog and cat. Oxford, UK: Butterworth Heinemann; 1992;2:142-151.

47. Todoroff RJ, Brodey RS. Oral and pharyngeal neoplasia in the dog: a retrospective survey of 361 cases. J Am Vet Med Assoc 1979;175:567-571.

48. Oakes MG, Lewis DD, Hedlund CS, Hosgood G. Canine oral neoplasia. Contin Educ Pract Vet Comp 1993;15:15-30.

49. Patnaik AK, Mooney S. Feline melanoma: a comparative study of ocular, oral and dermal neoplasms. Vet Pathol 1988;25:105-112.

50. Stebbins KE, Morse CC, Goldschmidt MH. Feline oral neoplasia: a ten-year survey. Vet Pathol 1989;26:121-128.

51. Miller WH, Scott DW, Anderson WI. Feline cutaneous melanocytic neoplasms: a retrospective analysis of 43 cases (1979-1991). Vet Dermatol 1993;4:19-26.

52. Engle CG, Brodey RS. A retrospective study of 395 feline neoplasms. J AM Anim Hosp Assoc 1969;5:21-31.

53. Day MJ, Lucke VM. Melanocytic neoplasia in the cat. J Small Anim Pract 1995;36:207-213.

54. Van Der- Linde SJS, De- Wit MML, Van- Garderen E, Molenbeek RF, Van DerVelde ZD, De- Weger RA. Cutaneous malignant melanomas in 57 cats: identification of (amelanotic) signet-ring and balloon cell types and verification of their origin by immunohistochemistry, electron microscopy, and in situ hybridization. Vet Pathol 1997;34:31-38.

55. Luna LD, Higginbottham ML, Henry CJ, Turnquist SE. Feline non-ocular melanoma: a retrospective study of 23 cases (1991-1999). J Feline Med Surg 2000; 2:173181. 
56. Weinborn AR, Issotta CC, Agurto MM, Lara LJ. Descripción clínica de hemangiosarcoma (HSA) cutáneo metastásico en un canino galgo: estudio clínico de un caso. Rev Med Vet 2015;2:107-116.

57. Aita N. Hemangioma of the ileum in a dog. J Vet Med Sci 2010;72:1071-1073.

58. López CC, Aréchiga OE, López CM. Protein kinases and transcription factors activation in response to UV-radiation of skin: Implications for carcinogenesis. Int J Mol Cienc 2012;13:142-172.

59. Duro ME, Campillos P, Causín S. El sol y los filtros solares. Medifam 2003;13: 159165.

60. Alvarez FE. Consecuencias del estrés oxidativo de la piel por radiaciones ultravioleta.http://scielo.sld.cu/scielo.php?script=sci_arttext\&pid=S0864030019950001 00004, Consultado 10 Nov, 2016.

61. Cruz CF. Fotosensibilización. http://www.ammveb.net/clinica/fotosensibilizacion. pdf, Consultado 20 Oct, 2016.

62. Fernández L, Poletta GL, Imhof A, Siroski PA. Efectos de la radiación ultravioleta natural y artificial (UVA/ UVB) sobre la concentración plasmática de calcio y fósforo y el crecimiento en crías de Caiman latirostris. InVet 2013;15:75-82.

63. Ross LI, Watson D, Escandarani S, Miranda A, Troncoso A. La radiación a la mesa. Rev Chilena Infect 2009;26:318-330. 